\title{
Some inequalities of the Turán type for confluent hypergeometric functions of the second kind
}

\author{
Feng Qi, Ravi Bhukya and Venkatalakshmi Akavaram
}

\begin{abstract}
In the paper, by virtue of the Hölder integral inequality, the authors derive some inequalities of the Turán type for confluent hypergeometric functions of the second kind, for the Mellin transforms, and for the Laplace transforms, and improve some known inequalities of the Turán type.
\end{abstract}

Mathematics Subject Classification (2010): 26D15, 26D20, 26D99, 33C15, 44A10, $44 \mathrm{~A} 15$.

Keywords: Inequality of the Turán type, confluent hypergeometric function of the second kind, improvement, Mellin transform, Laplace transform, Hölder integral inequality.

\section{Introduction}

In 1950, P. Turán [16] proved that the Legendre polynomials $P_{n}(x)$ satisfy

$$
P_{n}(x) P_{n+2}(x)-P_{n+1}^{2}(x) \leq 0
$$

for $|x| \leq 1$ and $n=0,1,2, \ldots$, where the equality holds only if $x= \pm 1$. An inequality of this kind is known as an inequality of the Turán type. This classical inequality has been extended to various special functions. For recent development on this classical inequality, please refer to $[2,3,4,6,7,11]$ and closely related reference therein.

It is known [1, p. 504-505] that confluent hypergeometric functions of the second kind $\psi(a, c, x)$ are also known as the Tricomi confluent hypergeometric functions, are a special solution of Kummer's differential equation

$$
x y^{\prime \prime}(x)+(c-x) y^{\prime}(x)-a y(x)=0,
$$

and have the integral representation

$$
\psi(a, c, x)=\frac{1}{\Gamma(a)} \int_{0}^{\infty} t^{a-1}(1+t)^{c-a-1} e^{-x t} \mathrm{~d} t
$$


for $a>0, c \in \mathbb{R}$, and $x>0$, where

$$
\Gamma(z)=\int_{0}^{\infty} t^{z-1} e^{-t} \mathrm{~d} t, \quad \Re(z)>0
$$

is the classical Euler gamma function [12, 13, 14, 15].

The Laplace transform and the Mellin transform of a function $f(t)$ are respectively defined by

$$
L(s)=\mathscr{L}(f)(s)=\int_{0}^{\infty} f(t) e^{-s t} \mathrm{~d} t, \quad s>0
$$

and

$$
M(s)=\mathscr{M}(f)(s)=\int_{0}^{\infty} f(t) t^{s-1} \mathrm{~d} t .
$$

These transforms are widely-used integral transforms with many applications in physics and engineering.

In this paper, we will study some Turán type inequalities for confluent hypergeometric functions of the second kind $\psi(a, c, x)$.

\section{Inequalities of the Turán type}

We are now in a position to find some inequality of the Turán type for confluent hypergeometric functions of the second kind. These newly-founded inequalities improve existed inequality of the Turán type in [4, Theorem 2].

Theorem 2.1. For $x>0, a>0$, and $c \in \mathbb{R}$, we have

$$
\psi^{2}(a+1, c, x)<\frac{a+1}{a} \psi(a, c, x) \psi(a+2, c, x) .
$$

Proof. The equality (1.1) can be reformulated as

$$
f(a) \triangleq \psi(a, c, x) \Gamma(a)=\int_{0}^{\infty}\left(\frac{t}{1+t}\right)^{a}(1+t)^{c-1} e^{-x t} \frac{1}{t} \mathrm{~d} t .
$$

Replacing $a$ by $\alpha p+(1-\alpha) q$ for $p, q>0, p \neq q$, and $\alpha \in(0,1)$ and using the well-known Hölder integral inequality give

$$
\begin{aligned}
f(\alpha p+(1-\alpha) q) & =\int_{0}^{\infty}\left(\frac{t}{1+t}\right)^{\alpha p+(1-\alpha) q}(1+t)^{c-1} e^{-x t} \frac{1}{t} \mathrm{~d} t \\
& =\int_{0}^{\infty}\left[t^{p-1}(1+t)^{c-p-1} e^{-x t}\right]^{\alpha}\left[t^{q-1}(1+t)^{c-q-1} e^{-x t}\right]^{1-\alpha} \\
& <\left[\int_{0}^{\infty} t^{p-1}(1+t)^{c-p-1} e^{-x t}\right]^{\alpha}\left[\int_{0}^{\infty} t^{q-1}(1+t)^{c-q-1} e^{-x t}\right]^{1-\alpha} \\
& =f^{\alpha}(p) f^{1-\alpha}(q) .
\end{aligned}
$$

This implies that the function $f$ is strictly logarithmically convex on $(0, \infty)$. Consequently, taking $\alpha=\frac{1}{2}, p=a$, and $q=a+2$ leads to $f^{2}(a+1)<f(a) f(a+2)$ which is equivalent to (2.1). The proof of Theorem 2.1 is complete. 
Theorem 2.2. For $x>0,0<a_{1}, a_{2}<a$, and $c \in \mathbb{R}$, we have

$$
\psi^{2}(a, c, x)<\frac{\Gamma\left(a_{1}\right) \Gamma\left(a_{2}\right)}{\Gamma^{2}(a)} \psi\left(a_{1}, c, x\right) \psi\left(a_{2}, c, x\right) .
$$

Proof. We continue to adopt the notation $f(a)$ in the proof of Theorem 2.1. Then

$$
\begin{aligned}
f^{\prime}(a) & =\frac{\mathrm{d}}{\mathrm{d} a}\left[\int_{0}^{\infty}\left(\frac{t}{1+t}\right)^{a}(1+t)^{c-1} \frac{1}{t} e^{-x t} \mathrm{~d} t\right] \\
& =\int_{0}^{\infty}\left(\frac{t}{1+t}\right)^{a} \ln \left(\frac{t}{1+t}\right)(1+t)^{c-1} \frac{1}{t} e^{-x t} \mathrm{~d} t \\
& <0 .
\end{aligned}
$$

Since $\ln \left(\frac{t}{1+t}\right)<0$ for $t>0$, the function $f(a)$ is decreasing on $(0, \infty)$ with respect to $a$. Accordingly, for $0<a_{1}, a_{2}<a$, we have $f(a)<f\left(a_{1}\right)$ and $f(a)<f\left(a_{2}\right)$. Consequently, it follows that $f^{2}(a)<f\left(a_{1}\right) f\left(a_{2}\right)$ which is equivalent to $(2.2)$. The proof of Theorem 2.2 is complete.

Theorem 2.3. For $x>0, a>0$, and $c_{1}, c_{2}<c \in \mathbb{R}$, we have

$$
\psi(a, c, x) \psi(a, c-1, x)<\psi^{2}(a, c+1, x)<\psi(a, c, x) \psi(a, c+2, x)
$$

and

$$
\psi\left(a, c_{1}, x\right) \psi\left(a, c_{2}, x\right)<\psi^{2}(a, c, x) .
$$

Proof. A straightforward computation yields

$$
\begin{aligned}
\psi^{\prime}(c) & =\frac{1}{\Gamma(a)} \frac{\mathrm{d}}{\mathrm{d} c}\left[\int_{0}^{\infty} t^{a-1}(1+t)^{c-a-1} e^{-x t} \mathrm{~d} t\right] \\
& =\frac{1}{\Gamma(a)} \int_{0}^{\infty} t^{a-1}(1+t)^{c-a-1} \ln (1+t) e^{-x t} \mathrm{~d} t \\
& >0 .
\end{aligned}
$$

This means that the function $\psi(a, c, x)$ is increasing with respect to $c \in \mathbb{R}$. Hence, for $c_{1}, c_{2}<c$, it follows that $\psi\left(a, c_{1}, x\right)<\psi(a, c, x)$ and $\psi\left(a, c_{2}, x\right)<\psi(a, c, x)$. Consequently, we obtain the inequality (2.4).

Taking $c_{1}=c-1$ and $c_{2}=c$ and replacing $c$ by $c+1$ in (2.4) deduce that

$$
\psi(a, c-1, x) \psi(a, c, x)<\psi^{2}(a, c+1, x) .
$$

From this inequality and the inequality $\psi^{2}(a, c+1, x)<\psi(a, c, x) \psi(a, c+2, x)$ in the paper [4], the inequality (2.3) follows immediately. The proof of Theorem 2.3 is complete.

Theorem 2.4. For $x, y>0, a>0, p, q>0$ such that $\frac{1}{p}+\frac{1}{q}=1$, and $c \in \mathbb{R}$, we have

$$
\psi\left(a, c, \frac{x}{p}+\frac{y}{q}\right)<\psi^{1 / p}(a, c, x) \psi^{1 / q}(a, c, y) .
$$


Proof. Applying the well-known Hölder integral inequality to the third variable $x$ in $\psi(a, c, x)$ arrives at

$$
\begin{aligned}
\psi(a, c & \left., \frac{x}{p}+\frac{y}{q}\right)=\frac{1}{\Gamma(a)} \int_{0}^{\infty} t^{a-1}(1+t)^{c-a-1} e^{-(x / p+y / q) t} \mathrm{~d} t \\
& =\frac{1}{\Gamma(a)} \int_{0}^{\infty}\left[t^{a-1}(1+t)^{c-a-1} e^{-x t}\right]^{1 / p}\left[t^{a-1}(1+t)^{c-a-1} e^{-y t}\right]^{1 / q} \\
& <\left[\frac{1}{\Gamma(a)} \int_{0}^{\infty} t^{a-1}(1+t)^{c-a-1} e^{-x t}\right]^{1 / p}\left[\frac{1}{\Gamma(a)} \int_{0}^{\infty} t^{q-1}(1+t)^{c-q-1} e^{-y t}\right]^{1 / q} \\
& =\psi^{1 / p}(a, c, x) \psi^{1 / q}(a, c, x) .
\end{aligned}
$$

Therefore, the inequality (2.5) is proved. The proof of Theorem 2.4 is complete.

Theorem 2.5. For $x, y>1$ such that $\frac{1}{x}+\frac{1}{y} \leq 1, a>0, p, q>0$ such that $\frac{1}{p}+\frac{1}{q}=1$, and $c \in \mathbb{R}$, we have

$$
\psi\left(a, c, \frac{x^{p}}{p}+\frac{y^{q}}{q}\right)<\psi^{1 / p}(a, c, p x) \psi^{1 / q}(a, c, q y) .
$$

Proof. Applying Young's inequality to the third variable $x$ in $\psi(a, c, x)$ results in

$$
\begin{aligned}
\psi\left(\frac{x^{p}}{p}+\frac{y^{q}}{q}\right)= & \frac{1}{\Gamma(a)} \int_{0}^{\infty} t^{a-1}(1+t)^{c-a-1} e^{-\left(x^{p} / p+y^{q} / q\right) t} \mathrm{~d} t \\
\leq & \frac{1}{\Gamma(a)} \int_{0}^{\infty} t^{a-1}(1+t)^{c-a-1} e^{-x y t} \\
\leq & {\left[\frac{1}{\Gamma(a)} \int_{0}^{\infty} t^{a-1}(1+t)^{c-a-1} e^{-p x t}\right]^{1 / p} } \\
& \times\left[\frac{1}{\Gamma(a)} \int_{0}^{\infty} t^{q-1}(1+t)^{c-q-1} e^{-q y t}\right]^{1 / q} \\
= & \psi^{1 / p}(a, c, p x) \psi^{1 / q}(a, c, q x) .
\end{aligned}
$$

The inequality (2.6) is thus proved. The proof of Theorem 2.5 is complete.

Theorem 2.6. For $x, y>0, a>0$, and $c \in \mathbb{R}$, we have

$$
\psi^{2}(a, c, x+y)<\psi(a, c, x) \psi(a, c, y) .
$$

For $x>0,0<y<1, a>0$, and $c \in \mathbb{R}$, we have

$$
\psi(a, c, x+y)<\psi(a, c, x y) .
$$

Proof. It is easy to see that the function $\psi(a, c, x)$ is decreasing with respect to $x \in$ $(0, \infty)$. Since $x<x+y$ and $y<x+y$, it follows that $\psi(a, c, x+y)<\psi(a, c, x)$ and $\psi(a, c, x+y)<\psi(a, c, y)$. This means the inequality $(2.7)$.

Similarly, the inequality (2.8) follows readily. The proof of Theorem 2.6 is complete. 


\section{Inequalities of the Turán type for the Mellin transform}

Now we discover an inequality of the Turán type for the Mellin transform.

Theorem 3.1. For $s>0$, the Mellin transform $M(s)$ satisfies

$$
F^{2}(s+1) \leq F(s) F(s+2) .
$$

Proof. Applying the Hölder integral inequality finds that

$$
\begin{aligned}
M(\alpha p+(1-\alpha) q) & =\int_{0}^{\infty} f(t) t^{\alpha p+(1-\alpha) q-1} \mathrm{~d} t \\
& =\int_{0}^{\infty}\left[f(t) t^{p-1}\right]^{\alpha}\left[f(t) t^{q-1}\right]^{1-\alpha} \mathrm{d} t \\
& \leq\left[\int_{0}^{\infty} f(t) t^{p-1} \mathrm{~d} t\right]^{\alpha}\left[\int_{0}^{\infty} f(t) t^{q-1} \mathrm{~d} t\right]^{1-\alpha} \\
& =M^{\alpha}(p) M^{1-\alpha}(q) .
\end{aligned}
$$

This means that the Mellin transform $M(s)$ is strictly logarithmically convex on $(0, \infty)$. Further letting $\alpha=\frac{1}{2}, p=s$, and $q=s+2$ in the above inequality leads to the inequality (3.1). The proof of Theorem 3.1 is complete.

Example 3.2. Entry 17.43 .26 in [9] states that

$$
M_{1}(s)=\mathscr{M}(\operatorname{cosech}(x))=2\left(1-2^{-s}\right) \Gamma(s) \zeta(s), \quad s>1 .
$$

By Theorem 3.1, it follows readily that

$$
M_{1}^{2}(s+1) \leq M_{1}(s) M_{1}(s+2) .
$$

After some simplification we acquire

$$
\zeta^{2}(s+1) \leq\left(\frac{s+1}{s}\right)\left[\frac{\left(1-2^{-s}\right)\left(1-2^{-s-2}\right)}{\left(1-2^{-s-1}\right)^{2}}\right] \zeta(s) \zeta(s+2), \quad s>1
$$

which improves the Turán type inequality for the zeta function in [11].

Example 3.3. Entry 6.3 .8 in [10] states that

$$
M_{2}(s)=\mathscr{M}\left(e^{-a x}\left(1-e^{-x}\right)^{-1}\right)=\Gamma(s) \zeta(s, a), \quad s>0, \quad a>0 .
$$

By Theorem 3.1, we derive

$$
M_{2}^{2}(s+1) \leq M_{2}(s) M_{2}(s+2) .
$$

After some simplification we acquire

$$
\zeta^{2}(s+1, a) \leq \frac{s+1}{s} \zeta(s, a) \zeta(s+2, a), \quad s>1, \quad a>0 .
$$

When $a=1$ in (3.2), we recover the Turán type inequality in [11]. 


\section{Inequalities of the Turán type for the Laplace transform}

Finally we find out an inequality of the Turán type for the Laplace transform.

Theorem 4.1. The Laplace transform $L(s)$ satisfies

$$
L^{2}(s+1) \leq L(s) L(s+2), \quad s>0 .
$$

Proof. By the Hölder integral inequality, we have

$$
\begin{aligned}
L(\alpha p+(1-\alpha) q) & =\int_{0}^{\infty} f(t) e^{-(\alpha p+(1-\alpha) q) t} \mathrm{~d} t \\
& =\int_{0}^{\infty}\left[f(t) e^{-p t}\right]^{\alpha}\left[f(t) e^{-q t}\right]^{1-\alpha} \mathrm{d} t \\
& \leq\left[\int_{0}^{\infty} f(t) e^{-p t} \mathrm{~d} t\right]^{\alpha}\left[\int_{0}^{\infty} f(t) e^{-q t} \mathrm{~d} t\right]^{1-\alpha} \\
& =L^{\alpha}(p) L^{1-\alpha}(q) .
\end{aligned}
$$

In other words, the Laplace transform $L(s)$ is strictly logarithmically convex on $(0, \infty)$. Specially, setting $\alpha=\frac{1}{2}, p=s$, and $q=s+2$ in the above inequality leads to (4.1). The proof of Theorem 4.1 is complete.

Example 4.2. Entry 4.15.29 in [10] states that

$$
L_{3}(s)=\mathscr{L}\left(\left(1-e^{-t}\right)^{\nu / 2} J_{\nu}\left(a\left(1-e^{-t}\right)^{1 / 2}\right)\right)=\Gamma(s)\left(\frac{2}{a}\right)^{s} J_{\nu+s}(a)
$$

for $s>0, a>0, \nu>-1$, where $J_{\mu}(z)$ denotes Bessel's functions. By Theorem 4.1, it follows that

$$
L_{3}^{2}(s+1) \leq L_{3}(s) L_{3}(s+2)
$$

which can be reformulated as

$$
J_{\nu+s+1}^{2}(a) \leq \frac{s+1}{s} J_{\nu+s}(a) J_{\nu+s+2}(a)
$$

for $s>0,1>a>0$, and $\nu>-\frac{1}{2}$.

When taking $\nu=0$ and replacing $s$ by $s-1$ for $s \geq 1$ in (4.2), we derive an upper bound of the Turán type inequality in [5, Eq. (2.3)] for $0<a<1$.

Example 4.3. Entry 4.3.11 in [10] reads that

$$
L_{4}(s)=\mathscr{L}\left(t^{2}-a^{2}\right)^{\nu-1 / 2}=\frac{1}{\sqrt{\pi}} \Gamma\left(\nu+\frac{1}{2}\right)\left(\frac{2 a}{s}\right)^{\nu} K_{\nu}(a s)
$$

for $s, a>0$ and $\nu>-\frac{1}{2}$, where $K_{\mu}(z)$ denotes modified Bessel's functions. By Theorem 4.1 , it follows that

$$
L_{4}^{2}(s+1) \leq L_{4}(s) L_{4}(s+2)
$$

which can be rewritten as

$$
K_{\nu}^{2}(a(s+1)) \leq\left[\frac{s^{2}+2 s+1}{s(s+2)}\right]^{\nu} K_{\nu}(a s) K_{\nu}(a(s+2))
$$

for $s, a>0$ and $\nu>-\frac{1}{2}$. 
Remark 4.4. Many other Turán type inequalities can be obtained for functions whose Laplace and Mellin transforms exists. In particular, we can prove some Turán type inequalities for the gamma, beta, extended beta, hypergeometric, error, and compliment error functions.

Remark 4.5. This paper is a slightly revised version of the preprint [8].

Acknowledgements. The authors appreciate anonymous reviewers for their valuable comments on the original version of this paper.

\section{References}

[1] Abramowitz, M., Stegun, I.A. (eds.), Handbook of Mathematical Functions with Formulas, Graphs, and Mathematical Tables, National Bureau of Standards, Applied Mathematics Series, 55, 10th printing, Washington, 1972.

[2] Baricz, Á., Turán type inequalities for hypergeometric functions, Proc. Am. Math. Soc., 136(2008), no. 9, 3223-3229;

Available online at https://doi.org/10.1090/S0002-9939-08-09353-2.

[3] Baricz, Á., Turán type inequalities for modified Bessel functions, Bull. Aust. Math. Soc., 82(2010), no. 2, 254-264;

Available online at https://doi.org/10.1017/S000497271000002X.

[4] Baricz, Á., Ismail, M.E.H., Turán type inequalities for Tricomi confluent hypergeometric functions, Constr. Approx., 37(2013), no. 2, 195-221;

Available online at https://doi.org/10.1007/s00365-012-9171-1.

[5] Baricz, Á., Pogány, T.K., Turán determinants of Bessel functions, Forum Math., 26(2014), no. 1, 295-322;

Available online at https://doi.org/10.1515/form.2011.160.

[6] Baricz, Á., Ponnusamy, S., On Turán type inequalities for modified Bessel functions, Proc. Amer. Math. Soc., 141(2013), no. 2, 523-532;

Available online at https://doi.org/10.1090/S0002-9939-2012-11325-5.

[7] Baricz, Á., Ponnusamy, S., Singh, S., Turán type inequalities for confluent hypergeometric function of second kind, Studia Sci. Math. Hungar., 53(2016), no. 1, 74-92;

Available online at https://doi.org/10.1556/012.2016.53.1.1330.

[8] Bhukya, R., Akavaram, V., Qi, F., Some inequalities of the Turán type for confluent hypergeometric functions of the second kind, HAL archives (2018);

Available online at https://hal.archives-ouvertes.fr/hal-01701854.

[9] Gradshteyn, I.S., Ryzhik, I.M., Table of Integrals, Series, and Products, Translated from the Russian, Translation Edited and With a Preface by Daniel Zwillinger and Victor Moll, Eighth Edition, Revised from the Seventh Edition, Elsevier/Academic Press, Amsterdam, 2015;

Available online at https://doi.org/10.1016/B978-0-12-384933-5.00013-8.

[10] Erdélyi, A., Magnus, W., Oberhettinger, F., Tricomi, F.G., Tables of Integral Transforms, Vol. I, McGraw-Hill Book Company, Inc., New York-Toronto-London, 1954.

[11] Laforgia, A., Natalini, P., Turán type inequalities for some special functions, J. Inequal. Pure Appl. Math., 7(2006), no. 1, Art. 22;

Available online at http://www.emis.de/journals/JIPAM/article638.html. 
[12] Qi, F., Bhukya, R., Akavaram, V., Inequalities of the Grünbaum type for completely monotonic functions, Adv. Appl. Math. Sci., 17(2018), no. 3, 331-339.

[13] Qi, F., Guo, B.-N., Complete monotonicity of the divided differences of di- and trigamma functions and its applications, Georgian Math. J., 23(2016), no. 2, 279-291; Available online at http://dx.doi.org/10.1515/gmj-2016-0004.

[14] Qi, F., Guo, B.-N., Integral representations and complete monotonicity of remainders of the Binet and Stirling formulas for the gamma function, Rev. R. Acad. Cienc. Exactas Fís. Nat. Ser. A Math. RACSAM, 111 (2017), no. 2, 425-434; Available online at https://doi.org/10.1007/s13398-016-0302-6.

[15] Qi, F., Li, W.-H., A logarithmically completely monotonic function involving the ratio of gamma functions, J. Appl. Anal. Comput., 5(2015), no. 4, 626-634; Available online at http://dx.doi.org/10.11948/2015049.

[16] Turán, P., On the zeros of the polynomials of Legendre, Časopis Pěst. Mat. Fys., 75(1950), 113-122.

Feng Qi

(1) College of Mathematics, Inner Mongolia University for Nationalities Tongliao 028043, Inner Mongolia, China;

(2) School of Mathematical Sciences, Tianjin Polytechnic University

Tianjin 300387, China;

(3) Institute of Mathematics, Henan Polytechnic University

Jiaozuo 454010, Henan, China

e-mail: qifeng618@gmail.com, qifeng618@hotmail.com

Ravi Bhukya

Department of Mathematics, Government College for Men

Kurnool, Andhra Pradesh, India

e-mail: ravidevi19@gmail.com

Venkatalakshmi Akavaram

Department of Mathematics, University College of Technology

Osmania University, Hyderrabad, Telangana, India

e-mail: akavaramvlr@gmail.com 